\title{
Treatment Outcomes of Transperitoneal Laparoscopic Adrenalectomy in Patients with Functional Adrenal Gland Tumors
}

\author{
Kriangsak Jenwitheesuk ${ }^{1}$, Kamonwan Jenwitheesuk ${ }^{2}$, Suriya Punchai ${ }^{3}$, Jakrapan Wittayapairoch ${ }^{4}$
}

\begin{abstract}
Background: Functional adrenal gland tumor is a tumor that presents with abnormal producing excess aldosterone hormone and affects the functionality of renin angiotensin aldosterone system which can be treated by laparoscopic adrenalectomy. The adrenal surgery can be approached in many techniques, such as transperitoneal and retroperitoneal approach.

Materials and methods: The inclusion criterion was all patients who had diagnosed with functional adrenal gland tumor underwent laparoscopic adrenalectomy since 2011-2017 at the Srinagarind Hospital, the Faculty of Medicine, Khon Kaen University, Thailand. Patients with a lesion more than $8 \mathrm{~cm}$ and patients with suspected metastatic lesions were excluded. Baseline clinical features, results of clinical evaluation, pathology, operative reports, and outcome of treatment were recorded.

Results: Seventy patients were treated with transperitoneal laparoscopic adrenalectomy. There were 18 men and 52 women. Mean age was $46.30 \pm 11.88$ years. All patients were presented with hypertension and 7 cases were presented with hypokalemia. Major histologic finding was adenoma. The mean operating time was $99.29 \pm 45.66$ minutes, estimated blood loss $82.36 \pm 202.14 \mathrm{~mL}$, and mean postoperative length of stay was $3.80 \pm 2.34$ days. Complications were occurred in six patients.

Conclusion: Transperitoneal laparoscopic surgery had good outcomes for functional adrenal gland tumor.

Keywords: Functional adrenal gland tumor, Transperitoneal approach, Transperitoneal laparoscopic adrenalectomy, Treatment outcomes.

World Journal of Endocrine Surgery (2019): 10.5005/jp-journals-10002-1258
\end{abstract}

\section{INTRODUCTION}

Functional adrenal gland tumor is a tumor that presents with abnormal producing excess aldosterone hormone and affects the functionality of renin angiotensin aldosterone system. The patients often come with high blood pressure symptoms and hypokalemia. The laparoscopic surgery is considered a replacement procedure for a major treatment in the surgical conditions as a transition from the open abdominal surgery to the laparoscopic surgery, which is more developed in both surgical techniques and surgical equipment. At present, when the development of the surgery is more prosperous, the surgeon began to see the advantages of laparoscopic surgery, especially from gallbladder surgery, which has the advantage to reduce the duration of the hospital, reducing pain, the patients can return to work quickly, and lower hospital costs. In addition, the treatment results are safely procedures and not different outcome from the open surgery. As a result, this surgical procedure is increasingly popular including laparoscopic adrenalectomy.

Laparoscopic adrenalectomy was first reported by Gagner et al. ${ }^{1}$ The adrenal surgery can be approached in many techniques, such as transperitoneal, transthoracic, or retroperitoneal approach. ${ }^{2,3}$ But, favorable procedures include transperitoneal and retroperitoneal approach. The purpose of this research is to provide an overview of the basic characteristics of functional adrenal gland tumor, which is important in choosing the patient for surgery, as well as the result from the therapy of the transperitoneal laparoscopic adrenalectomy.

\section{Materials and Methods}

The inclusion criterion was all patients who had diagnosed with functional adrenal gland tumor underwent laparoscopic
1,3,4 Department of Surgery, General Surgery Unit, Faculty of Medicine, and Sleep Apnea Resaerch Group, Khon Kaen University, Khon Kaen, Thailand

${ }^{2}$ Department of Surgery, Plastic and Reconstructive Unit, Faculty of Medicine, Khon Kaen University, Thailand

Corresponding Author: Kriangsak Jenwitheesuk, Department of Surgery, General Surgery Unit, Faculty of Medicine, and Sleep Apnea Resaerch Group, Khon Kaen University, Khon Kaen, Thailand, Phone: +66-43-363542, e-mail: surgerykkh@gmail.com

How to cite this article: Jenwitheesuk $\mathrm{K}$, Jenwitheesuk $\mathrm{K}$, et al. Treatment Outcomes of Transperitoneal Laparoscopic Adrenalectomy in Patients with Functional Adrenal Gland Tumors. World J Endoc Surg 2019;11(2):41-45.

Source of support: Nil

Conflict of interest: None

adrenalectomy since 2011-2017 at the Srinagarind Hospital, the Faculty of Medicine, Khon Kaen University, Khon Kaen, Thailand. Patients with a lesion more than $8 \mathrm{~cm}$ and patients with suspected metastatic lesions were excluded. For laparoscopic adrenalectomy, the transperitoneal approach via the flank in the lateral decubitus was performed for all patients. Three-ports technique was used for the left-sided tumors and four-ports technique for the rightsided ones. Baseline clinical features, medical treatment, results of clinical evaluation, and the pathology and operative reports were recorded. For the outcome of treatment, the patients were also interviewed either by telephone or during follow-up time at the out-patient department for completely free or only improve symptoms after 6 months. 


\section{Surgical Technique of Transperitoneal Laparoscopic Adrenalectomy with Lateral Flank Approach}

The patient's posture preparation is an important step for the key to success. After the anesthetic processes were completed, all patients had inserted nasogastric tube and retained urinary catheterization. For arterial line was necessary in the case of pheochromocytoma. After positioning the patient in the lateral decubitus, the bed should be adjusted to a reverseTrendelenburg position in order to help with more workspace and make the blood or liquid lower down from the surgical field. For the operating sites, left adrenalectomy is suitable with three-ports technique as well as four-ports technique for right adrenalectomy.

The general principle of the adrenal gland surgery means of extracapsular dissection without using the tools is to catch the adrenal glands, to avoid unnecessary injuries to the organ, and to prevent tissue's breakdown, which can cause tumor cells spreading. The opening of the workspace can be done by pushing or lifting the adrenal glands with a dull grip, or catching the periadrenal fat. The main step is to stop bleeding with the periadrenal area in order to see the apparent anatomy not endangers the surrounding organs. Adrenal vein is a blood vessel that must be handled first, especially in the pheochromocytoma which reduces catecholamines. For the small blood vessels, ultrasonic coagulator can be used.

The left adrenalectomy starts from the separate of transverse colon and splenic flexure colon and by the dissection of splenocolic ligamint and the white line of Toldt. After that, release the lateral attachment and diaphragmatic attachment to the middle of the spleen which also presents the tail of the pancreas and the superomedial part of adrenal gland.

At the top of the upper pole of the kidney, starting from the inferior margin by finding the adrenal vein first, it should always be tried according to the confluence of renal vein. Be careful to demonstrate renal artery and renal vein after that. It needs to clip adrenal vein 1-2 clips on the adrenal gland side and 3 clips on the proximal side. Between the dissection of the superior pole of adrenal gland, accessory adrenal vein from the inferior phrenic vein may be found. To secure the adrenal artery, electrocauterization, harmonic scalpel, or LigaSure can be used. To remove the tumor from the abdomen, sterile plastic bag can be used.

The procedure on the right adrenal gland is quite similar to the left. There is difference by more than one port of the retractor. This port is used by lifting the liver up during which the corner of the retractor must be parallel to the lower liver edge. The process of dissections is starts from the release of the right triangular ligament, which can make the right lobe liver fall on the medial side and use fan retractor placed through xiphoid port push liver on top. Afterward, it will see adrenal gland on the right near retrohepatic inferior vena cava (IVC) without needing to separate colon or duodenum. The surgeons have to divide peritoneum which covers the adrenal gland on the lateral to IVC and then dissect from the top to the lower area until demonstrating the adrenal vein connected to the IVC. This vein mostly presents at the superomedial position of the adrenal gland. Right adrenal vein is short, so it needs 3 clips at IVC side and 2 clips at gland side. After that, the surgeons release the gland from medial to the lateral until completed free and remove the tumor through the sterile plastic bag.

\section{Results}

Seventy patients were treated with transperitoneal laparoscopic adrenalectomy between 2011 and 2017 at the Srinagarind Hospital. There were 18 men and 52 women (Table 1). Mean age was $46.30 \pm$ 11.88 years. All patients were presented with hypertension and 7 cases were presented with hypokalemia. Major histologic finding was adenoma which is about $87.14 \%$, followed by $10 \%$ adrenal hyperplasia, and $2.86 \%$ pheochromocytoma. Predominant side was left side in $62.86 \%$. For the 70 patients, only five cases (7.14\%) required conversion to open surgery, mainly caused by intra-abdominal organ injury in the first twenty cases of learning curve. The mean operating time was $99.29 \pm 45.66$ minutes, estimated blood loss $82.36 \pm 202.14 \mathrm{~mL}$, and mean postoperative length of stay was $3.80 \pm 2.34$ days. Complications were occurred in six patients (Table 2).

Table 1: Baseline clinical features and clinical outcome of transperitoneal laparoscopic adrenalectomy

\begin{tabular}{ll}
\hline Clinical data & No. of patients (\%) \\
\hline Gender & \\
Male & $18(25.41)$ \\
Female & $52(74.29)$ \\
Mean age & $46.30 \pm 11.88$ \\
Mean tumor size (cm) & $2.4 \pm 1.53$ \\
Tumor side & \\
Right & $26(37.14)$ \\
Left & $44(62.86)$ \\
Mean operating time (minute) & $99.29 \pm 45.66$ \\
Mean blood loss (mL) & $82.36 \pm 202.14$ \\
Hospital stay (day) & $3.80 \pm 2.34$ \\
Pathological diagnosis & \\
Adenoma & $61(87.14)$ \\
Adrenal hyperplasia & $7(10)$ \\
Pheochromocytoma & $2(2.86)$ \\
Conversion to open surgery & $5(7.14)$ \\
Complication & $6(8.57)$ \\
Outcome & \\
Completely free of symptoms & $46(65.71)$ \\
after 6 months & \\
Improve by reducing the doses of & $24(34.29)$ \\
antihypertensive drugs & \\
Persist hypertension or hypokalemia & 0 \\
\hline &
\end{tabular}

Table 2: Postoperative morbidity

\begin{tabular}{ll}
\hline Postoperative morbidity & No. of patients \\
\hline Duodenal injury and subphrenic & 1 \\
collection & 1 \\
Tear liver & 1 \\
Not seen right adrenal gland & 1 \\
Surgical site infection & 1 \\
Left renal vein tear & 1 \\
Splenic flexure colon tear & \\
\hline
\end{tabular}




\section{Discussion}

Laparoscopic adrenalectomy for functional adrenal gland tumor is a popular surgical technique including transperitoneal and retroperitoneal approach. ${ }^{4}$ By transperitoneal approach, it has many advantages: $:^{5-7}$ (I) it is a wide operative field. (II) If the add-on tool is needed, it can easily be done. (III) The function requires a distance of the tool, so the surgery in the abdomen has a wide space. (IV) The surgeon is familiar with the anatomical characteristics, make a short learning curve. (V) It enables easy control of the adrenal vein with minimal gland manipulation. (VI) It can handle the bleeding easily if there is trauma to the blood vessels. In terms of disadvantages, it may increase the risk of intra-abdominal adhesion. In the case of retroperitoneal approach, ${ }^{8,9}$ it is a method that can be done without through the intra-abdomen and avoid adhesion. It can also be done if the patient has the abdominal surgery history but transperitoneal approach may be difficult. It provides a direct approach to the adrenal gland, and the bilateral adrenalectomy can be performed during a single procedure. Disadvantages are less space in the operation and limited space tools. There were comparisons that try to measure the treatment results of both methods from the systematic review ${ }^{10}$ on 22 studies which found that retroperitoneal approach has the same surgical effect as transperitoneal approach, but the hospital length may be shorter. Selection of surgical methods, however, depends on the aptitude of each surgeon. From the review of the literature on laparoscopic adrenalectomy, it showed that the results of two approaches had a difference. The shortest operative time was $32.33 \pm 7.05$ minutes and the longest was $176 \pm 58$ minutes. Blood loss was between $16.25 \pm$ 9.78 to $107 \pm 119$ cc. The rate of conversion from laparoscopic surgery to open surgery was $0-7 \%$. Complication rate was $0-19 \%$. There was only one report for one death. ${ }^{11}$ Completely free of symptoms were $28-62.22 \%$.

A systematic review and meta-analysis demonstrated that hypertension cure rate of unilateral adrenalectomy ranged between $15 \%$ and $96 \% .{ }^{12}$ Our study demonstrated a complete cure rate of about $65.71 \%$, operative time of $99.29 \pm 45.66$ minutes, estimated blood loss of $82.36 \pm 202.14 \mathrm{cc}$, range of postoperative hospital stay of about $3.80 \pm 2.34$ days which all were the favorable outcome when compared with other studies (Tables 3 and 4). For surgical technique, we limited the exact size of the tumor not more than $8 \mathrm{~cm}$, which is the standard safety procedure to avoid the morbidity. ${ }^{13}$ In addition, there was a retrospective study in patients with adrenal tumors that were greater than or equal to 6 centimeters, and the diagnostic imaging finding was incompatible with cancer; laparoscopic adrenalectomy could be safe. ${ }^{14}$

For pheochromocytoma, there was no contraindication for laparoscopic adrenalectomy but a more complicated surgical procedure due to the characteristic of enrich blood supply..$^{15}$ Also, when the gland was manipulated, blood pressure fluctuates more. Work coordination with the anesthetist made our procedure a success. In these tumors, posterior retroperitoneal approach reduces the duration of surgery, blood loss, and hospital stay. ${ }^{16}$ Fortunately, there was a small number for pheochromocytoma. The almost coincidence of this series represented the adenoma which the same like others. ${ }^{14,17,18}$ For hemostasis control technique, Harmonic scalpel was used for dissection during surgery in less than $5 \mathrm{~mm}$ blood vessels. It could decrease the operative time and secure the procedure.

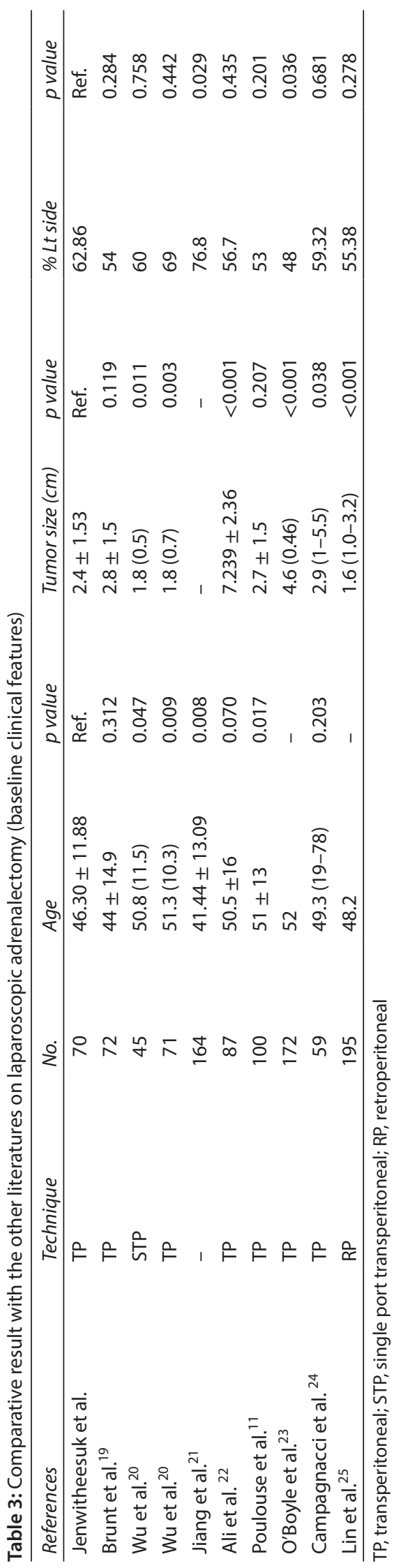




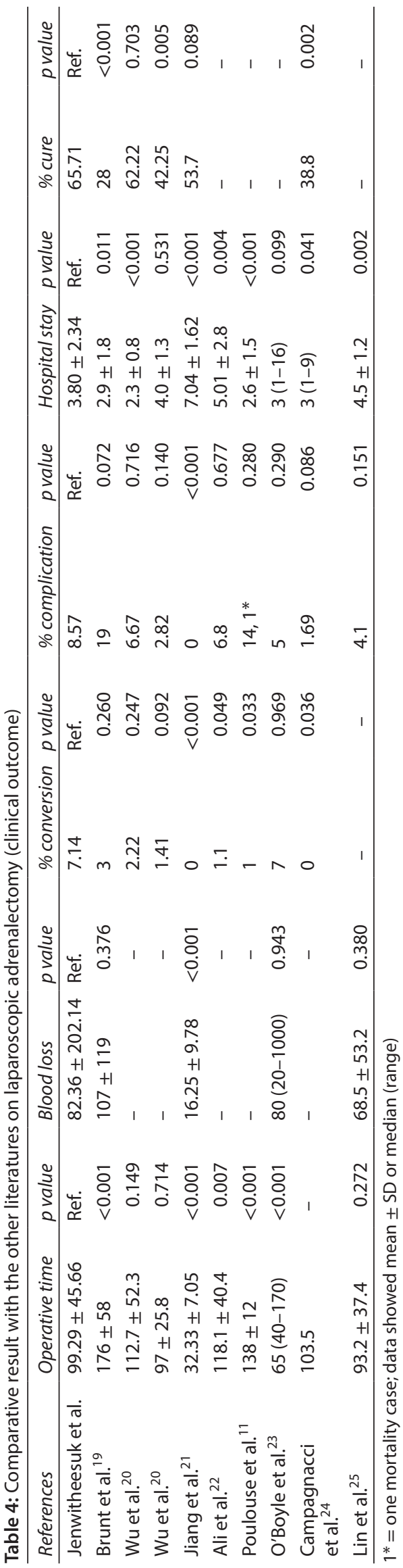

\section{Conclusion}

Transperitoneal laparoscopic surgery has good outcomes for functional adrenal gland tumor that replaces open surgery, as it is a safe way, lower morbidity, short period of surgical time, and quick recovery.

\section{ACKnOWLedgment}

The authors would like to thank Sleep Apnea Research Group, Research Center in Back, Neck and Other Joint Pain and Human Performance, and Research and Training Center for Enhancing Quality of Life of Working Age People, Khon Kaen University for their kind support.

\section{References}

1. Gagner M, Lacroix A, et al. Laparoscopic adrenalectomy in Cushing's syndrome and pheochromocytoma. N Engl J Med 1992 Oct;327(14):1033. DOI: 10.1056/NEJM199210013271417.

2. Del Pizzo JJ. Transabdominal laparoscopic adrenalectomy. Curr Urol Rep 2003 Feb;4(1):81-86. DOI: 10.1007/s11934-003-0064-5.

3. Mellon MJ, Sethi A, et al. Laparoscopic adrenalectomy: Surgical techniques. Indian J Urol 2008 Oct-Dec;24(4):583-589. DOI: 10.4103/0970-1591.44277.

4. Nakagawa K, Murai M. Laparoscopic adrenalectomy: current status with a review of Japanese literature. Biomed Pharmacother 2002;56(1):107s-112s. DOI: 10.1016/S0753-3322(02)00232-9.

5. Lezoche $E$, Guerrieri M, et al. Perioperative results of 214 laparoscopic adrenalectomies by anterior transperitoneal approach. Surg Endosc 2008 Feb;22(2):522-526. DOI: 10.1007/s00464-007-9555-7.

6. Paganini AM, Guerrieri M, et al. Management of adrenal incidentaloma by laparoscopic transperitoneal anterior and submesocolic approach. Langenbecks Arch Surg 2016 Feb;401(1):71-79. DOI: 10.1007/s00423015-1367-y.

7. Conzo G, Tartaglia E, et al. Minimally invasive approach for adrenal lesions: Systematic review of laparoscopic versus retroperitoneoscopic adrenalectomy and assessment of risk factors for complications. Int J Surg 2016 Apr;28(1):S118-S123. DOI: 10.1016/j.ijsu.2015.12.042.

8. Gasman D, Droupy S, et al. Laparoscopic adrenalectomy: the retroperitoneal approach. J Urol 1998 Jun;159(6):1816-1820. DOI: 10.1016/S0022-5347(01)63163-X.

9. Chai YJ, Woo JW, et al.Comparative outcomes of lateral transperitoneal adrenalectomy versus posterior retroperitoneoscopic adrenalectomy in consecutive patients: a single surgeon's experience. Asian J Surg 2016 Apr;39(2):74-80. DOI: 10.1016/j.asjsur.2015.04.005.

10. Constantinides VA, Christakis I, et al. Systematic review and metaanalysis of retroperitoneoscopic versus laparoscopic adrenalectomy. Br J Surg 2012 Dec;99(12):1639-1648. DOI: 10.1002/bjs.8921.

11. Poulose BK, Holzman MD, et al. Laparoscopic adrenalectomy: 100 resections with clinical long-term followup. Surg Endosc 2005 Mar;19(3):379-385. DOI: 10.1007/s00464-004-8914-x.

12. Zhou $Y$, Zhang M, et al. Hypertension outcomes of adrenalectomy in patients with primary aldosteronism: a systematic review and meta-analysis. BMC Endocr Disord 2017 Oct;17(1):61. DOI: 10.1186/ s12902-017-0209-z.

13. Gupta PK, Natarajan B, et al. Outcomes after laparoscopic adrenalectomy. Surg Endosc 2011 Mar;25(3):784-794. DOI: 10.1007/ s00464-010-1256-y.

14. Palazzo FF, Sebag F, et al. Long-term outcome following laparoscopic adrenalectomy for large solid adrenal cortex tumors. World J Surg 2006 May;30(5):893-898. DOI: 10.1007/s00268-005-0288-2.

15. Gagner M, Pomp A, et al. Laparoscopic adrenalectomy: lessons learned from 100 consecutive procedures. Ann Surg 1997 Sep;226(3):238-246. DOI: 10.1097/00000658-199709000-00003.

16. Dickson PV, Alex GC, et al. Posterior retroperitoneoscopic adrenalectomy is a safe and effective alternative to transabdominal 
laparoscopic adrenalectomy for pheochromocytoma. Surgery 2011 Sep;150(3):452-458. DOI: 10.1016/j.surg.2011.07.004.

17. Li H, Liu J, et al. Favorable surgical outcomes of aldosteroneproducing adenoma based on lateralization by CT imaging and hypokalemia: a non-AVS-based strategy. Int Urol Nephrol 2017 Dec;49(12):2151-2156. DOI: 10.1007/s11255-017-1705-9.

18. Christakis I, Livesey JA, et al. Laparoscopic Adrenalectomy for Conn's Syndrome is Beneficial to Patients and is Cost Effective in England. J Invest Surg 2017 May;12:1-7.

19. Brunt LM, Moley JF, et al. Outcomes analysis in patients undergoing laparoscopic adrenalectomy for hormonally active adrenal tumors. Surgery 2001 Oct;130(4):629-634. DOI: 10.1067/msy.2001. 116920.

20. Wu CH, Er LK, et al. Is Laparoendoscopic Single-Site Adrenalectomy a Feasible Alternative in Treating Aldosterone-Producing Adenoma? Biomed Res Int 2016;2016:6894381.
21. Jiang SB, Guo XD, et al. A retrospective study of laparoscopic unilateral adrenalectomy for primary hyperaldosteronism caused by unilateral adrenal hyperplasia. Int Urol Nephrol 2014 Jul;46(7):1283-1288. DOI: 10.1007/s11255-013-0614-9.

22. Ali JM, Liau SS, et al. Laparoscopic adrenalectomy: auditing the 10 year experience of a single centre. Surgeon 2012 Oct;10(5):267-272. DOI: 10.1016/j.surge.2011.08.003.

23. O'Boyle CJ, Kapadia CR, et al. Laparoscopic transperitoneal adrenalectomy. Surg Endosc 2003 Dec;17(12):1905-1909. DOI: 10.1007/ s00464-002-8878-7.

24. Campagnacci $R$, Crosta $F$, et al. Long-term results of laparoscopic adrenalectomy for primary aldosteronism. J Endocrinol Invest 2009 Jan;32(1):57-62. DOI: 10.1007/BF03345680.

25. Lin $Y$, Li L, et al. Experience of retroperitoneoscopic adrenalectomy in 195 patients with primary aldosteronism. Int J Urol 2007 Oct;14(10):910-913. DOI: 10.1111/j.1442-2042.2007.01860.x. 